K. Sato and M. Yamazato

Nagoya Math. J.

Vol. 97 (1985), 71-94

\title{
COMPLETELY OPERATOR-SELFDECOMPOSABLE DISTRIBUTIONS AND OPERATOR-STABLE DISTRIBUTIONS
}

\author{
KEN-ITI SATO AND MAKOTO YAMAZATO
}

\section{§1. Introduction}

Urbanik introduces in [16] and [17] the classes $L_{m}$ and $L_{\infty}$ of distributions on $R^{1}$ and finds relations with stable distributions. Kumar-Schreiber [6] and Thu [14] extend some of the results to distributions on Banach spaces. Sato [7] gives alternative definitions of the classes $L_{m}$ and $L_{\infty}$ and studies their properties on $R^{d}$. Earlier Sharpe [12] began investigation of operator-stable distributions and, subsequently, Urbanik [15] considered the operator version of the class $L$ on $R^{d}$. Jurek [3] generalizes some of Sato's results [7] to the classes associated with one-parameter groups of linear operators in Banach spaces. Analogues of Urbanik's classes $L_{m}$ (or $L_{\infty}$ ) in the operator case are called multiply (or completely) operatorselfdecomposable. They are studied in relation with processes of OrnsteinUhlenbeck type or with stochastic integrals based on processes with homogeneous independent increments (Wolfe [18], [19], Jurek-Vervaat [5], Jurek [2], [4], and Sato-Yamazato [9], [10]). The purpose of the present paper is to continue the preceding papers, to give explicit characterizations of completely operator-selfdecomposable distributions and operatorstable distributions on $R^{d}$, and to establish relations between the two classes. For this purpose we explore the connection of the structures of these classes with the Jordan decomposition of a basic operator $Q$.

Let $\mathscr{P}\left(R^{d}\right)$ be the class of probability distributions on $R^{d}$, and $M_{+}\left(R^{d}\right)$ be the class of linear operators on $R^{d}$ all of whose eigenvalues have positive real parts. Let

$$
t^{Q}=\sum_{n=0}^{\infty}(n !)^{-1}(\log t)^{n} Q^{n} \quad \text { for } t>0
$$

Received September 1, 1983. 
For $H \subset \mathscr{P}\left(R^{a}\right)$ and $Q \in M_{+}\left(R^{d}\right)$, let $\mathscr{L}_{Q}(H)$ be the class of probability distributions $\mu$ on $R^{d}$ such that there are independent $R^{a}$-valued random variables $X_{n}, a_{n} \in R^{d}$, and $t_{n}>0$ satisfying

(i) the distribution of $X_{n}$ belongs to $H$,

(ii) the distribution of $t_{n}^{Q} \sum_{j=1}^{n} X_{j}-a_{n}$ converges to $\mu$ as $n \rightarrow \infty$,

(iii) for every $\varepsilon>0, \max _{1 \leq j \leq n} P\left(\left|t_{n}^{Q} X_{j}\right|>\varepsilon\right) \rightarrow 0$ as $n \rightarrow \infty$.

Define

$$
\begin{aligned}
& O L_{0}\left(R^{d}, Q\right)=\mathscr{L}_{Q}\left(\mathscr{P}\left(R^{d}\right)\right), \\
& O L_{m}\left(R^{d}, Q\right)=\mathscr{L}_{Q}\left(O L_{m-1}\left(R^{d}, Q\right)\right) \quad \text { for } m=1,2, \cdots, \\
& O L_{\infty}\left(R^{d}, Q\right)=\bigcap_{0 \leq m<\infty} O L_{m}\left(R^{d}, Q\right) .
\end{aligned}
$$

Let $O S\left(R^{d}, Q\right)$ be the class of probability distributions $\mu$ on $R^{d}$ such that there are independent identically distributed $X_{n}, a_{n} \in R^{a}$, and $t_{n}>0$ that satisfy (ii). We denote $O L_{m}\left(R^{d}, Q\right), O L_{\infty}\left(R^{d}, Q\right)$ and $O S\left(R^{d}, Q\right)$ shortly by $L_{m}(Q), L_{\infty}(Q)$, and $S(Q)$, respectively. Let $I D\left(R^{d}\right)$ be the class of infinitely divisible distributions on $R^{d}$. Let $I$ be the identity operator. We use the notation $(T \mu)(E)=\mu\left(T^{-1}(E)\right)$ for a linear operator $T$. The characteristic function of $\mu$ is denoted by $\hat{\mu}(z)$. For $\mu \in I D\left(R^{d}\right)$ and $t>0, \mu^{t}$ denotes the distribution with characteristic function $\hat{\mu}(z)^{t}$. The delta distribution at a point $a$ is denoted by $\delta_{a}$. The following characterizations are known.

Proposition 1.1 (Sato [7] for $Q=I$, Jurek [3] for general $Q$, see also Sato-Yamazato [10]). Let $0 \leq m<\infty$. A distribution $\mu$ belongs to $L_{m}(Q)$ if and only if, for every $t \in(0,1)$, there exists $\mu_{t}$ in $L_{m-1}(Q)$ such that

$$
\mu=t^{Q} \mu * \mu_{t} \text {. }
$$

Here we understand $L_{-1}(Q)=\mathscr{P}\left(R^{d}\right)$.

Proposition 1.2 (Sharpe [12] and Jurek [3]). A distribution $\mu$ belongs to $S(Q)$ if and only if $\mu \in I D\left(R^{d}\right)$ and there exists some $\alpha>0$ such that, for every $t>0$,

$$
\mu^{t^{\alpha}}=t^{Q} \mu * \delta_{a(t)}
$$

with some $a(t) \in R^{d}$.

By Proposition 1.1, a distribution in $L_{0}(Q)$ is called $Q$-selfdecomposable. The property (1.2) in Proposition 1.2 is said as $\mu$ is operator-stable with exponent $\alpha^{-1} Q$. We call distributions in $L_{m}(Q) m+1$ times $Q$-selfdecomposable, and call distributions in $L_{\infty}(Q)$ completely $Q$-selfdecomposable. 
If $\mu$ is in $L_{\infty}(Q)$ for some $Q \in M_{+}\left(R^{d}\right)$, then $\mu$ is called completely operatorselfdecomposable. It is easy to see that

$$
I D \supset L_{0}(Q) \supset L_{1}(Q) \supset \cdots \supset L_{\infty}(Q) \supset S(Q),
$$

and each of $L_{m}(Q), 0 \leq m<\infty$, and $L_{\infty}(Q)$ is closed under convolution, convergence, and raising to the $t$-th power.

\section{§2. Condition for complete operator-selfdecomposability}

We use the following notations throughout: $\langle x, y\rangle$ is the Euclidean inner product in $R^{d}$ or the Hermitian inner product in $C^{d},|x|$ denotes $\langle x, x\rangle^{1 / 2}$, and $\mathscr{B}\left(R^{d}\right)$ is the class of Borel sets in $R^{d}$. The adjoint operator of a linear operator $T$ is denoted by $T^{\prime}$. For a real symmetric (or Hermitian) operator $A, \phi_{A}(z)$ stands for $\langle A z, z\rangle$, the quadratic (or Hermitian) form associated with $A$. Further,

$$
\begin{aligned}
& g(z, x)=e^{i\langle x, z\rangle}-1-i\langle x, z\rangle\left(1+|x|^{2}\right)^{-1}, \\
& h(s)=s^{2}\left(1+s^{2}\right)^{-1} .
\end{aligned}
$$

Let $Q \in M_{+}\left(R^{d}\right)$. There are constants $c_{j}>0(1 \leq j \leq 4)$ such that

$$
\begin{aligned}
& c_{4} u^{c_{2}}|x| \leq\left|u^{Q} x\right| \leq c_{3} u^{c_{1}}|x| \quad \text { for } 0<u \leq 1, \\
& c_{3}^{-1} u^{c_{1}}|x| \leq\left|u^{Q} x\right| \leq c_{4}^{-1} u^{c_{2}}|x| \quad \text { for } u \geq 1 .
\end{aligned}
$$

Denote by $S_{Q}$ the set of $\xi \in R^{d}$ such that $|\xi|=1$ and $\left|u^{Q} \xi\right|>1$ for every $u>1$. Every $x \neq 0$ in $R^{d}$ is uniquely expressed as $x=u^{Q} \xi, \xi \in S_{Q}, u>0$.

An infinitely divisible distribution $\mu$ has the Lévy representation $(\gamma$, $A, \nu)$, that is,

$$
\hat{\mu}(z)=\exp \left(i\langle\gamma, z\rangle-2^{-1} \phi_{A}(z)+\int_{R^{d}} g(z, x) \nu(d x)\right), \quad z \in R^{d},
$$

where $\gamma$ is in $R^{d}, A$ is real symmetric nonnegative definite, and $\nu$ is a measure on $R^{d}$ satisfying $\nu(\{0\})=0$ and $\int h(|x|) \nu(d x)<\infty$. The measure $\nu$ is the Lévy measure of $\mu$. The operator $A$ is the Gaussian covariance operator of $\mu$. We call $\mu$ purely non-Gaussian if $A=0$. If $\gamma=0$ and $A=0$, then we call $\mu$ centered purely non-Gaussian. If $\gamma=0$ and $\nu=0$, then $\mu$ is called centered Gaussian. The class of $\mu \in I D\left(R^{d}\right)$ such that its Lévy measure $\nu$ satisfies $\int_{|x|>1} \log |x| \nu(d x)<\infty$ is denoted by $I D\left(R^{d}\right)_{\log }$.

The class of $Q$-selfdecomposable distributions on $R^{d}$ is characterized 
by properties of $A$ and $\nu$. In Sato-Yamazato [9] the following is called the first representation $\left(\gamma, A, \lambda, k_{\xi}(u)\right)$ of $\mu \in L_{0}(Q)$.

Proposition 2.1. Let $Q \in M_{+}\left(R^{d}\right)$. If $\mu \in L_{0}(Q)$, then

$$
\hat{\mu}(z)=\exp \left(i\langle\gamma, z\rangle-2^{-1} \phi_{A}(z)+\int_{S_{Q}} \lambda(d \xi) \int_{0}^{\infty} g\left(z, u^{Q} \xi\right) k_{\xi}(u) u^{-1} d u\right),
$$

where $\gamma \in R^{d}, A$ is nonnegative definite,

$$
\phi_{A}\left(e^{-t Q^{\prime}} z\right) \text { is nonincreasing in } t>0 \text { for every } z \in R^{d},
$$

$\lambda$ is the zero measure or a probability measure on $S_{Q}, k_{\xi}(u)$ is measurable in $\xi$, nonnegative, right-continuous, nonincreasing in $u$ and

$$
0<\int_{0}^{\infty} h\left(\left|u^{Q} \xi\right|\right) k_{\xi}(u) u^{-1} d u=c<\infty
$$

with $c$ independent of $\xi$. These $\gamma, A$, and $\lambda$ are uniquely determined by $\mu$ and $k_{\xi}(u)$ is uniquely determined by $\mu$ up to $\xi$ of $\lambda$-measure zero. Conversely, given $\gamma, A, \lambda$, and $k_{\xi}(u)$ with the above properties, one can find $\mu$ in $L_{0}(Q)$ satisfying (2.4).

Remark. 2.1. In [9], the property (2.5) is expressed in another form. Each of the following two conditions is equivalent to the condition (2.5):

$$
\begin{gathered}
\phi_{A}(z)=\int_{0}^{\infty} \phi_{B}\left(e^{-t Q^{\prime}} z\right) d t \text { with some nonnegative definite } B ; \\
Q A+A Q^{\prime} \text { is nonnegative definite . }
\end{gathered}
$$

In fact, (2.7) implies (2.5) since

$$
\phi_{A}\left(e^{-t Q^{\prime}} z\right)=\int_{t}^{\infty} \phi_{B}\left(e^{-s Q^{\prime}} z\right) d s .
$$

The condition (2.5) implies (2.8) and (2.8) implies (2.7), because

$$
(-d / d t)\left(\phi_{A}\left(e^{-t Q^{\prime}} z\right)\right)=\phi_{Q A+A Q^{\prime}}\left(e^{-t Q^{\prime}} z\right) .
$$

Note that the integral in (2.7) is finite by virtue of (2.1).

Let us give a characterization of completely $Q$-selfdecomposable distributions. This generalizes results of Urbanik [16], Sato [7] and Jurek [3].

Theorem 2.1. Let $Q \in M_{+}\left(R^{a}\right)$. A measure $\mu$ belongs to $L_{\infty}(Q)$ if and only if $\mu \in L_{0}(Q)$ and $A$ and $k_{\xi}(u)$ in its first representation satisfy the following: 
(2.9) $\phi_{A}\left(e^{-t Q^{\prime}} z\right)$ is completely monotone in $t>0$ for every $z \in R^{d}$,

(2.10) $\quad k_{\xi}\left(e^{t}\right)$ is completely monotone in $-\infty<t<\infty$ for $\lambda$-almost every $\xi \in S_{Q}$.

Proof. If $\mu \in L_{0}(Q)$, then we can find a unique $\mu_{0} \in I D\left(R^{d}\right)_{\log }$ such that

$$
\hat{\mu}(z)=\exp \int_{0}^{\infty} \log \hat{\mu}_{0}\left(e^{-t Q^{\prime}} z\right) d t .
$$

The mapping $\Psi_{Q}$ defined by $\Psi_{Q} \mu=\mu_{0}$ is one-to-one and onto (Jurek [2] and Sato-Yamazato [9]). Let $(r, A, \nu)$ and $(a, B, \rho)$ be the Lévy representations of $\mu$ and $\mu_{0}$, respectively. Let $\left(\gamma, A, \lambda, k_{\xi}(u)\right)$ be the first representation of $\mu$. Then we have

$$
\begin{aligned}
& (-d / d t)\left(\phi_{A}\left(e^{-t Q^{\prime}} z\right)\right)=\phi_{B}\left(e^{-t Q^{\prime}} z\right), \\
& \nu(E)=\int_{S_{Q}} \lambda(d \xi) \int_{0}^{\infty} \chi_{E}\left(u^{Q} \xi\right) k_{\xi}(u) u^{-1} d u=\int_{R^{d}} \rho(d x) \int_{0}^{\infty} \chi_{E}\left(e^{-t Q} x\right) d t, \\
& \rho(E)=-\int_{S_{Q}} \lambda(d \xi) \int_{0}^{\infty} \chi_{E}\left(u^{Q} \xi\right) d k_{\xi}(u) \quad \text { for } E \in \mathscr{B}\left(R^{d}\right),
\end{aligned}
$$

where $\chi_{E}$ is the indicator function of $E$ (Sato-Yamazato [9]). For $1 \leq m$ $<\infty$, it can be proved that $\mu \in L_{m}(Q)$ if and only if $\Psi_{Q} \mu \in L_{m-1}(Q)$ (Jurek [4] and Sato-Yamazato [10]).

Suppose that $\mu \in L_{\infty}(Q)$. Then we can operate $\Psi_{Q}$ on $\mu$ as many times as we like. Thus we obtain

$$
(-d / d t)^{m}\left(\phi_{A}\left(e^{-t Q^{\prime}} z\right)\right)=\phi_{B_{m}}\left(e^{-t Q^{\prime}} z\right)
$$

with some nonnegative definite $B_{m}$, hence (2.9). Suppose $\nu \neq 0$. Then $\rho \neq 0$. Let $\left(a, B, \lambda^{0}, k_{\xi}^{0}(u)\right)$ be the first representation of $\mu_{0}$. Then

$$
\rho(E)=\int_{s_{Q}} \lambda^{0}(d \xi) \int_{0}^{\infty} \chi_{E}\left(u^{Q} \xi\right) k_{\xi}^{0}(u) u^{-1} d u .
$$

Hence we have

$$
\begin{gathered}
k_{\xi}\left(u_{1}\right)-k_{\xi}\left(u_{2}\right)=b(\xi) \int_{u_{1}}^{u_{2}} k_{\xi}^{0}(u) u^{-1} d u \quad \text { for } 0<u_{1}<u_{2}, \\
\lambda^{0}(d \xi)=b(\xi) \lambda(d \xi),
\end{gathered}
$$

where

$$
b(\xi)=-\int_{0}^{\infty} h\left(\left|u^{Q} \xi\right|\right) d k_{\xi}(u)\left(\int_{R^{d}} h(|x|) \rho(d x)\right)^{-1} .
$$

It follows that 


$$
(-d / d t)\left(k_{\xi}\left(e^{t}\right)\right)=b(\xi) k_{\xi}^{0}\left(e^{t}\right) \geq 0 .
$$

Repeating this, we see that (2.10) holds.

Conversely, suppose that $\mu \in L_{0}(Q)$ satisfying the conditions (2.9) and (2.10). Then $\phi_{B}\left(e^{-t Q^{\prime}} z\right)$ is also completely monotone. We define $k_{\xi}^{0}(u)$ and $\lambda^{0}$ by the above formulas. Then we see that $\hat{\mu}_{0}(z)$ has the representation (2.4) with $a, B, \lambda^{0}$, and $k_{\xi}^{0}(u)$ in place of $\gamma, A, \lambda$, and $k_{\xi}(u)$, and that $k_{\xi}^{0}\left(e^{t}\right)$ is completely monotone in $t$. Hence $\mu_{0} \in L_{0}(Q)$, that is, $\mu \in L_{1}(Q)$, and, moreover, $B$ and $k_{\xi}^{0}(u)$ satisfy the conditions (2.9) and (2.10). Repeating this argument, we see $\mu \in L_{m}(Q)$ for every $m$.

Remark 2.2. The argument above shows that if $\mu \in L_{\infty}(Q)$ then $\phi_{A}\left(e^{-t Q^{\prime}} z\right)$ is completely monotone not only in $0<t<\infty$, but also in $-\infty<t<\infty$.

\section{§3. Gaussian completely operator-selfdecomposable distributions- Complex characterization}

We consider Gaussian distributions on $R^{d}$. Let $Q \in M_{+}\left(R^{d}\right)$. Since $L_{\infty}(Q)$ is closed under translation, we can restrict our attention to centered Gaussian distributions. The structure of $L_{\infty}(Q)$ heavily depends on the Jordan decomposition of $Q$. So it is convenient to use the complex $d$ dimensional space $C^{d}$. A linear operator on $R^{d}$ and its unique extension to $C^{d}$ will be denoted by the same symbol. Thus $Q$ and $Q^{\prime}$ both act on $C^{d}$. Let $\left\{\alpha_{1}, \cdots, \alpha_{p}\right\}$ be the set of distinct eigenvalues of $Q$. Then $\left\{\bar{\alpha}_{1}, \cdots\right.$, $\left.\bar{\alpha}_{p}\right\}$ is the set of distinct eigenvalues of $Q^{\prime}$. Since $Q$ is real, $Q$ and $Q^{\prime}$ have the same set of eigenvalues. Let $f(\zeta)$ be the minimal polynomial of $Q$ (equivalently, of $Q^{\prime}$ ). That is, $f$ is the polynomial of the least degree with real coefficients, satisfying $f(Q)=0$, with coefficient 1 in the highest term. Decompose it into linear factors

$$
f(\zeta)=\left(\zeta-\alpha_{1}\right)^{n(1)} \cdots\left(\zeta-\alpha_{p}\right)^{n(p)},
$$

where $n(1), \cdots, n(p)$ are positive integers. Let

$$
V_{j}=\operatorname{Kernel}\left(Q-\alpha_{j} I\right)^{n(j)} \text { in } C^{d} \quad \text { for } 1 \leq j \leq p .
$$

Then, denoting the direct sum by $\oplus$, we have

$$
C^{d}=V_{1} \oplus \cdots \oplus V_{p} \text {. }
$$

Let $T_{j}$ be the projector of $C^{a}$ onto $V_{j}$ in the decomposition (3.2). Namely, $x=T_{1} x+\cdots+T_{p} x$ where $T_{j} x \in V_{j}$ for $1 \leq j \leq p$. Let 


$$
V_{j}^{\prime}=\operatorname{Kernel}\left(Q^{\prime}-\bar{\alpha}_{j} I\right)^{n(j)} \text { in } C^{d} \quad \text { for } 1 \leq j \leq p
$$

Then

$$
C^{d}=V_{1}^{\prime} \oplus \cdots \oplus V_{p}^{\prime}
$$

We see that (3.4) is the decomposition dual to (3.2). That is, $V_{j}^{\prime}$ and $V_{k}$ are orthogonal for $j \neq k$. The adjoint operator $T_{j}^{\prime}$ of $T_{j}$ is the projector of $C^{d}$ to $V_{j}^{\prime}$ in the decomposition (3.4). The following is a main result.

TheOREM 3.1. Let $\mu$ be a centered Gaussian distribution with covariance operator $A$. Then, $\mu \in L_{\infty}(Q)$ if and only if

$$
\left(Q-\alpha_{j}\right) A T_{j}^{\prime}=0 \quad \text { for } 1 \leq j \leq p .
$$

Remark 3.1. An alternative expression is that $\mu \in L_{\infty}(Q)$ if and only if

$$
\begin{array}{cc}
A\left(Q^{\prime}-\bar{\alpha}_{j}\right) T_{j}^{\prime}=0 & \text { for } 1 \leq j \leq p, \\
T_{k} A T_{j}^{\prime}=0 & \text { for } j \neq k .
\end{array}
$$

We need three lemmas.

Lemma 3.1. Let $z_{0} \in C^{d}$. If $A$ is nonnegative definite and $\phi_{A}\left(z_{0}\right)=0$, then $A z_{0}=0$.

Proof. For every $w \in C^{d}$ and real number $t$, we have

$$
0 \leq \phi_{A}\left(w+t z_{0}\right)=\phi_{A}(w)+2 t \operatorname{Re}\left\langle A z_{0}, w\right\rangle
$$

and hence $\operatorname{Re}\left\langle A z_{0}, w\right\rangle=0$. Also $\operatorname{Im}\left\langle A z_{0}, w\right\rangle=\operatorname{Re}\left\langle A z_{0}, i w\right\rangle=0$. Hence $\left\langle A z_{0}, w\right\rangle=0$ for every $w \in C^{d}$.

Lemma 3.2. Let $\alpha>0$ and $a, b, c$ real, and let $g(t)=e^{-\alpha t}(a+b t+$ $\left.c t^{2}\right)$. If $g(t)$ is completely monotone in $-\infty<t<\infty$, then $a \geq 0$ and $b=$ $c=0$.

Proof. It follows from $g \geq 0$ that $a \geq 0$ and $c \geq 0$. Suppose that $c>0$. Let

$$
g_{n}(t)=e^{-\alpha t}\left(a-(4 c)^{-1} b^{2}-n \alpha^{-2} c+c t^{2}\right), \quad n=0,1, \cdots .
$$

Since $g(t)=e^{\alpha b /(2 c)} g_{0}\left(t+(2 c)^{-1} b\right), g_{0}(t)$ is completely monotone. We see that $(-d / d t) g_{n-1}(t)=\alpha e^{-1} g_{n}\left(t-\alpha^{-1}\right)$. Hence, by induction, $g_{n}(t)$ is completely monotone. This implies

$$
a-(4 c)^{-1} b^{2}-n \alpha^{-2} c \geq 0 \quad \text { for } n=0,1, \cdots,
$$


which is absurd. It follows that $c=0$. Hence $b=0$.

Lemma 3.3. Let $\alpha$ and $\beta$ be distinct complex numbers with positive real parts. Let $a$ and $b$ real and $c$ be complex. Let

$$
g_{\zeta \eta}(t)=a \zeta \bar{\zeta} e^{-t(\alpha+\alpha)}+2 \operatorname{Re}\left(c \zeta \bar{\eta} e^{-t(\alpha+\bar{\beta})}\right)+b \eta \bar{\eta} e^{-t(\beta+\bar{\beta})} .
$$

If, for every choice of $\zeta, \eta \in C$, the function $g_{\zeta \eta}(t)$ is completely monotone in a neighborhood of 0 , then $a \geq 0, b \geq 0$, and $c=0$.

Proof. Nonnegativity of $(-d / d t)^{n} g_{\zeta \eta}(0)$ means that the matrix

$$
\left(\begin{array}{ll}
a(\alpha+\bar{\alpha})^{n} & \bar{c}(\bar{\alpha}+\beta)^{n} \\
c(\alpha+\bar{\beta})^{n} & b(\beta+\bar{\beta})^{n}
\end{array}\right)
$$

is a nonnegative definite Hermitian. Hence $a \geq 0, b \geq 0$, and

$$
a b(\alpha+\bar{\alpha})^{n}(\beta+\bar{\beta})^{n}-c \bar{c}(\alpha+\bar{\beta})^{n}(\bar{\alpha}+\beta)^{n} \geq 0 .
$$

Let $\alpha=\alpha_{1}+i \alpha_{2}$ and $\beta=\beta_{1}+i \beta_{2}$. Then

$$
(\alpha+\bar{\beta})(\bar{\alpha}+\beta)=\left(\alpha_{1}+\beta_{1}\right)^{2}+\left(\alpha_{2}-\beta_{2}\right)^{2}>4 \alpha_{1} \beta_{1}=(\alpha+\bar{\alpha})(\beta+\bar{\beta}),
$$

since $\alpha \neq \beta$. Hence we have $|c|^{2} \leq k^{n} a b$ with some $k$ satisfying $0<k<1$. Hence $c=0$.

Proof of Theorem 3.1. Suppose that $\mu \in L_{\infty}(Q)$. Let us prove (3.6). It is enough to show that, for every positive integer $n$,

$$
\left(Q^{\prime}-\bar{\alpha}_{j}\right)^{n} z_{0}=0 \quad \text { implies } A\left(Q^{\prime}-\bar{\alpha}_{j}\right) z_{0}=0 .
$$

We prove this by induction in $n$. If $n=1$, the assertion is trivial. Suppose that (3.8) is true for $n-1$ in place of $n$, and assume that $\left(Q^{\prime}-\bar{\alpha}_{j}\right)^{n} z_{0}$ $=0$. Then, $A\left(Q^{\prime}-\bar{\alpha}_{j}\right)^{\ell} z_{0}=0$ for $\ell \geq 2$. Let us write $\left(Q^{\prime}-\bar{\alpha}_{j}\right)^{\ell} z_{0}=z_{\ell}$ and $\alpha_{j}=\alpha$. Since

$$
e^{-t Q^{\prime}} z_{0}=e^{-t \alpha}\left(z_{0}-t z_{1}+2^{-1} t^{2} z_{2}+\cdots+((n-1) !)^{-1}(-t)^{n-1} z_{n-1}\right),
$$

we have

$$
A e^{-t Q^{\prime}} z_{0}=e^{-t \alpha}\left(A z_{0}-t A z_{1}\right) .
$$

Let $g(t)=\phi_{A}\left(e^{-t Q^{\prime}} z_{0}\right)$. We have

$$
g(t)=e^{-t(\alpha+\alpha)} \phi_{A}\left(z_{0}-t z_{1}\right)=e^{-t(\alpha+\alpha)}\left(\phi_{A}\left(z_{0}\right)-2 t \operatorname{Re}\left\langle A z_{0}, z_{1}\right\rangle+t^{2} \phi_{A}\left(z_{1}\right)\right) .
$$

Since $g(t)$ is completely monotone in $-\infty<t<\infty$ by Theorem 2.1 and Remark 2.2, we obtain $\phi_{A}\left(z_{1}\right)=0$ by Lemma 3.2. Hence $A z_{1}=0$ by Lemma 
3.1, which proves (3.8). Let us show the property (3.7). Let $z_{0} \in V_{j}^{\prime}, w_{0} \in$ $V_{k}^{\prime}$, and $j \neq k$. Write $\alpha_{j}=\alpha, \alpha_{k}=\beta,\left(Q^{\prime}-\bar{\alpha}\right)^{\ell} z_{0}=z_{\ell}$, and $(Q-\bar{\beta})^{\ell} w_{0}=w_{\ell}$. We have $A z_{\ell}=A w_{\ell}=0$ for $\ell \geq 1$ by (3.6). For $\zeta, \eta \in C$, let $g_{\zeta \eta}(t)=$ $\phi_{A}\left(e^{-t Q^{\prime}}\left(\zeta z_{0}+\eta w_{0}\right)\right)$. Using the expression of $e^{-t Q^{\prime}} z_{0}$ and $e^{-t Q^{\prime}} w_{0}$ analogous to (3.9), we get

$$
\begin{aligned}
g_{\zeta \eta}(t) & =\phi_{A}\left(e^{-t \bar{\zeta} \zeta z_{0}}+e^{-t \bar{\beta}} \eta w_{0}\right) \\
& =e^{-t(\alpha+\bar{\alpha})} \zeta \bar{\zeta} \phi_{A}\left(z_{0}\right)+2 \operatorname{Re}\left(e^{-t(\alpha+\beta)} \bar{\zeta}\left\langle A z_{0}, w_{0}\right\rangle\right)+e^{-t(\beta+\bar{\beta})} \eta \bar{\eta} \phi_{A}\left(w_{0}\right) .
\end{aligned}
$$

Since $g_{\zeta \eta}(t)$ is completely monotone, we get $\left\langle A z_{0}, w_{0}\right\rangle=0$ by Lemma 3.3. Thus we have (3.7). Now we have also the property (3.5). In fact, we see that, for any $z$ and $w$,

$$
\left\langle\left(Q-\alpha_{j}\right) A T_{j}^{\prime} z, w\right\rangle=\sum_{k=1}^{p}\left\langle T_{j}^{\prime} z, A\left(Q^{\prime}-\bar{\alpha}_{j}\right) T_{k}^{\prime} w\right\rangle=0,
$$

using (3.6) for $k=j$ and (3.7) for $k \neq j$.

Conversely, suppose that $A$ satisfies (3.5). Then we have (3.6) since $\phi_{A}\left(\left(Q^{\prime}-\bar{\alpha}_{j}\right) T_{j}^{\prime} z\right)=\left\langle\left(Q-\alpha_{j}\right) A\left(Q^{\prime}-\bar{\alpha}_{j}\right) T_{j}^{\prime} z, T_{j}^{\prime} z\right\rangle=0$, and we have (3.7) since (3.5) implies that $A T_{j}^{\prime}$ has range in $V_{j}$. By the Jordan decomposition of $Q^{\prime}$, there are vectors $z_{j \ell} \in V_{j}^{\prime}\left(1 \leq \ell \leq \ell_{j}\right)$ and nonnegative integers $n(j, \ell)$ such that $\left(Q^{\prime}-\bar{\alpha}_{j}\right)^{n(j, \ell)+1} z_{j \ell}=0$ and that the system $\left\{z_{j \ell n}=\right.$ $\left.\left(Q^{\prime}-\bar{\alpha}_{j}\right)^{n} z_{j \ell}: 1 \leq j \leq p, 1 \leq \ell \leq \ell_{j}, 0 \leq n \leq n(j, \ell)\right\}$ is a basis of $C^{d}$. For any given $z \in C^{d}$, we have

$$
z=\sum_{j, \ell, n} c_{j \ell n} z_{j \ell n} \quad \text { for some } c_{j \ell n} \in C
$$

and, hence,

$$
e^{-t Q^{\prime}} z=\sum_{j, \ell, n} e^{-t \overline{\alpha(j)}} c_{j \ell n} \sum_{m=0}^{n(j, \ell)-n}(m !)^{-1}(-t)^{m} z_{j, \ell, n+m}
$$

where $\alpha(j)=\alpha_{j}$. Therefore, by (3.6) and (3.7),

$$
\phi_{A}\left(e^{-t Q^{\prime}} z\right)=\sum_{j} e^{-t(\alpha(j)+\overline{\alpha(j)})} \phi_{A}\left(\sum_{\ell} c_{j \ell 0} z_{j \ell 0}\right),
$$

which is completely monotone. It follows from Theorem 2.1 that $\mu \in L_{\infty}(Q)$. The proof of Theorem 3.1 is complete.

\section{§4. Gaussian completely operator-selfdecomposable distributions- Real characterization}

We rewrite the characterization in Theorem 3.1 in a real form and, then, give a decomposition theorem of Gaussian distributions in $L_{\infty}(Q)$. 
Arrange the distinct eigenvalues of $Q$ in such a way that $\alpha_{1}, \cdots, \alpha_{q}$ are real and $\alpha_{q+1}, \cdots, \alpha_{p}$ are not real, $\alpha_{j}=\bar{\alpha}_{j+r}(q+1 \leq j \leq q+r)$, and $q+$ $2 r=p$. Here $q$ or $r$ may possibly be zero. Let $\beta_{j}$ and $\gamma_{j}$ be the real and the imaginary part of $\alpha_{j}$, respectively. The minimal polynomial of $Q^{\prime}$ is

$$
f(\zeta)=f_{1}(\zeta)^{n(1)} \cdots f_{q+r}(\zeta)^{n(q+r)},
$$

where $f_{j}(\zeta)=\zeta-\alpha_{j}=\zeta-\beta_{j}$ for $1 \leq j \leq q$ and $f_{j}(\zeta)=\left(\zeta-\beta_{j}\right)^{2}+\gamma_{j}^{2}$ for $q+1 \leq j \leq q+r$. Let

$$
W_{j}^{\prime}=\operatorname{Kernel} f_{j}\left(Q^{\prime}\right)^{n(j)} \quad \text { in } R^{d} \text { for } 1 \leq j \leq q+r .
$$

Then

$$
R^{d}=W_{1}^{\prime} \oplus \cdots \oplus W_{q+r}^{\prime}
$$

As in the proof of Theorem 3.1, let $z_{j l} \in V_{j}^{\prime}\left(1 \leq j \leq p, 1 \leq \ell \leq \ell_{j}\right)$ and $n(j, \ell) \geq 0$ be such that $\left(Q^{\prime}-\bar{\alpha}_{j}\right)^{n(j, \ell)+1} z_{j \ell}=0$ and the system

$$
\left\{z_{j \ell n}=\left(Q^{\prime}-\bar{\alpha}_{j}\right)^{n} z_{j \ell}: 1 \leq j \leq p, 1 \leq \ell \leq \ell_{j}, 0 \leq n \leq n(j, \ell)\right\}
$$

is a basis of $C^{a}$. For $1 \leq j \leq q$, we can choose $z_{j \ell}$ real so that $\left\{z_{j \ell n}: 1 \leq\right.$ $\left.\ell \leq \ell_{j}, 0 \leq n \leq n(j, \ell)\right\}$ is a basis of $W_{j}^{\prime}$. For $q+1 \leq j \leq q+r$, we have $\ell_{j}=\ell_{j+r}$ and $n(j, \ell)=n(j+r, \ell)$ and we can choose $z_{j \ell}$ and $z_{j+r, \ell}$ in such a way that $z_{j \ell}=\bar{z}_{j+r, \ell}$. Let $\xi_{j \ell n}$ and $\eta_{j \ell n}$ be the real and the imaginary part of $z_{j \ell n}$, respectively, for $q+1 \leq j \leq q+r$. The system $\left\{\xi_{j \ell n}, \eta_{j \ell n}: 1 \leq\right.$ $\left.\ell \leq \ell_{j}, 0 \leq n \leq n(j, \ell)\right\}$ is then a basis of $W_{j}^{\prime}$. The following theorem gives martrix representation of $A$ when these bases are used.

TheOREm 4.1. Let $\mu$ be centered Gaussian with covariance operator A. Then $\mu \in L_{\infty}(Q)$ if and only if the following four conditions are satisfied:

$$
\begin{gathered}
\phi_{A}\left(z_{j \ell n}\right)=0 \quad \text { for } 1 \leq j \leq q, 1 \leq \ell \leq \ell_{j}, n \geq 1 \\
\phi_{A}\left(\xi_{j \ell n}\right)=\phi_{A}\left(\eta_{j \ell n}\right)=0 \quad \text { for } q+1 \leq j \leq q+r, 1 \leq \ell \leq \ell_{j}, n \geq 1 \\
\left\langle A \xi_{j \ell 0}, \xi_{j m 0}\right\rangle=\left\langle A \eta_{j \ell 0}, \eta_{j m 0}\right\rangle \quad \text { and }\left\langle A \xi_{j \ell 0}, \eta_{j m 0}\right\rangle=-\left\langle A \eta_{j \ell 0}, \xi_{j m 0}\right\rangle \\
\text { for } q+1 \leq j \leq q+r, 1 \leq \ell \leq \ell_{j}, 1 \leq m \leq \ell_{j}(\ell=m \text { inclusive }) \\
\langle A z, w\rangle=0 \quad \text { for } z \in W_{j}^{\prime}, w \in W_{k}^{\prime}, 1 \leq j \leq q+r, 1 \leq k \leq q+r, j \neq k
\end{gathered}
$$

Proof. Suppose that $\mu \in L_{\infty}(Q)$. Then (4.4) and (4.7) follow directly from (3.6) and (3.7). The condition (4.5) also follows from (3.6) since $\xi_{j \ell n}$ and $\eta_{j \ell n}$ are linear combinations of $z_{j \ell n}$ and $z_{j+r, \ell, n}$. Since $\xi_{j \ell 0}+i \eta_{j \ell 0}=z_{j \ell 0}$ $\in V_{j}^{\prime}$ and $\xi_{j m 0}-i \eta_{j m 0}=\bar{z}_{j m 0} \in V_{j+r}^{\prime}$, we get (4.6) from (3.7), rewriting $\left\langle A z_{\text {jeo }}, \bar{z}_{j m 0}\right\rangle=0$. 
Conversely, suppose that the conditions (4.4)-(4.7) are satisfied. Then (3.6) follows from (4.4) and (4.5), because (3.6) is equivalent to that $\phi_{A}\left(z_{j \ell n}\right)$ $=0$ for $1 \leq j \leq p, 1 \leq \ell \leq \ell_{j}, n \geq 1$. For $1 \leq j \leq q$, the system

$$
\left\{z_{j \ell n}: 1 \leq \ell \leq \ell_{j}, 0 \leq n \leq n(j, \ell)\right\}
$$

is a basis of $V_{j}^{\prime}$ in $C^{d}$. For $q+1 \leq j \leq q+r$, the system $\left\{\xi_{j \ell n}, \eta_{j \ell n}: 1 \leq\right.$ $\left.\ell \leq \ell_{j}, 0 \leq n \leq n(j, \ell)\right\}$ is a basis of $V_{j}^{\prime} \oplus V_{j+r}^{\prime}$ in $C^{d}$. Hence (3.7) follows from (4.7) except in the case where $j \geq q+1$ and $k=j+r$. It remains to show that $\langle A z, w\rangle=0$ for $z=z_{j \ell n}$ and $w=\bar{z}_{j m s}$ when $q+1 \leq j \leq q$ $+r, 1 \leq \ell \leq \ell_{j}, 1 \leq m \leq \ell_{j}, n \geq 0$, and $s \geq 0$. If $n \geq 1$ or $s \geq 1$, then this is proved by (4.5). If $n=s=0$, then this is a consequence of (4.6). The proof is complete.

Before proceeding to examples we give a lemma.

Lemma 4.1. Let $T$ be an invertible linear operator on $R^{d}$. Then, $\mu \epsilon$ $L_{\infty}(Q)$ if and only if $T \mu \in L_{\infty}\left(T Q T^{-1}\right)$.

Proof. It suffices to prove that $\mu \in L_{m}(Q)$ if and only if $T \mu \in L_{m}\left(T Q T^{-1}\right)$. We can show this by induction, using Proposition 1.1. Note that the relation (1.1) is equivalent to

$$
T \mu=t^{T Q T-1} T \mu * T \mu_{t} \quad \text { for } 0<t<1 .
$$

Examples. If we change $Q$ to $T Q T^{-1}$ then $A$ changes to $T A T^{\prime}$ by Lemma 4.1. So we assume that $Q$ itself has the real Jordan canonical form. Let $d=2$. The form of $Q$ has the following four possibilities:

$$
\left(\begin{array}{ll}
\alpha & 0 \\
0 & \alpha
\end{array}\right),\left(\begin{array}{cc}
\alpha & 1 \\
0 & \alpha
\end{array}\right),\left(\begin{array}{cc}
\alpha_{1} & 0 \\
0 & \alpha_{2}
\end{array}\right),\left(\begin{array}{cc}
\beta & -\gamma \\
\gamma & \beta
\end{array}\right)
$$

where $\alpha, \alpha_{1}, \alpha_{2}$, and $\beta$ are positive, $\alpha_{1} \neq \alpha_{2}$, and $\gamma \neq 0$. The necessary and sufficient condition for a symmetric, nonnegative definite matrix $A$ to be a covariance matrix of a Gaussian distribution in $L_{\infty}(Q)$ is that $A$ has, respectively, the form

$$
\left(\begin{array}{ll}
a & c \\
c & b
\end{array}\right),\left(\begin{array}{ll}
a & 0 \\
0 & 0
\end{array}\right),\left(\begin{array}{ll}
a & 0 \\
0 & b
\end{array}\right),\left(\begin{array}{ll}
a & 0 \\
0 & a
\end{array}\right)
$$

Similarly we can determine the form of $A$ for $L_{\infty}(Q)$ in higher dimensions. Write $J(a, b)=\left(\begin{array}{cc}a & -b \\ b & a\end{array}\right)$. Let us consider, for $d=3$ or 4 , four typical cases where $Q$ has the form 


$$
\left(\begin{array}{lll}
\alpha & 1 & 0 \\
0 & \alpha & 1 \\
0 & 0 & \alpha
\end{array}\right),\left(\begin{array}{lll}
\alpha & 1 & 0 \\
0 & \alpha & 0 \\
0 & 0 & \alpha
\end{array}\right),\left(\begin{array}{ll}
J(\beta, \gamma) & J(0,0) \\
J(0,0) & J(\beta, \gamma)
\end{array}\right),\left(\begin{array}{ll}
J(\beta, \gamma) & J(1,0) \\
J(0,0) & J(\beta, \gamma)
\end{array}\right) .
$$

Here $\alpha>0, \beta>0$, and $\gamma \neq 0$. In the respective case, the form of $A$ is as follows:

$$
\left(\begin{array}{lll}
a & 0 & 0 \\
0 & 0 & 0 \\
0 & 0 & 0
\end{array}\right),\left(\begin{array}{lll}
a & 0 & c \\
0 & 0 & 0 \\
c & 0 & b
\end{array}\right),\left(\begin{array}{ll}
J(a, 0) & J(c, d) \\
J(c,-d) & J(b, 0)
\end{array}\right),\left(\begin{array}{ll}
J(a, 0) & J(0,0) \\
J(0,0) & J(0,0)
\end{array}\right) .
$$

In order to formulate the next theorem, let

$$
W_{j}=\operatorname{Kernel} f_{j}(Q)^{n(j)} \quad \text { in } R^{d} \text { for } 1 \leq j \leq q+r
$$

where $f_{j}(\zeta), 1 \leq j \leq q+r$, are the polynomials of degree 1 or 2 in (4.1). Then

$$
R^{d}=W_{1} \oplus \cdots \oplus W_{q+r} .
$$

This is the decomposition dual to (4.3). Let $U_{j}$ be the projector of $R^{d}$ onto $W_{j}$ in the decomposition (4.9). Namely, $x=U_{1} x+\cdots+U_{q+r} x$ where $U_{j} x \in W_{j}$ for $1 \leq j \leq q+r$. The adjoint operator $U_{j}^{\prime}$ of $U_{j}$ is the projector onto $W_{j}^{\prime}$ in the decomposition (4.3). For $q+1 \leq j \leq q+r$, recall that $\alpha_{j}=\bar{\alpha}_{j+r}$; it is easy to see that $V_{j}=\bar{V}_{j+r}$ and that $T_{j} x=\overline{T_{j+r} x}$ and $U_{j} x=T_{j} x+T_{j+r} x$ for $x \in R^{d}$. For $1 \leq j \leq q$, we have $U_{j} x=T_{j} x$ for $x \in$ $R^{d}$. Let

$$
N_{j}=\operatorname{Kernel} f_{j}(Q) \quad \text { in } R^{d} \text { for } 1 \leq j \leq q+r .
$$

Theorem 4.2. Suppose that $\mu$ is a centered Gaussian distribution in $L_{\infty}(Q)$. Then, Spt $\mu$ is a Q-invariant linear subspace of $R^{d}$ and the minimal polynomial of the restriction of $Q$ to Spt $\mu$ does not have double roots. There exists a unique decomposition

$$
\mu=\mu_{1} * \cdots * \mu_{q+r},
$$

where each $\mu_{j}$ is a centered Gaussian distribution such that Spt $\mu_{j} \subset W_{j}$. Moreover, $\mu_{j} \in L_{\infty}(Q)$ and $\operatorname{Spt} \mu_{j} \subset N_{j}$.

We need a simple lemma. Proof is omitted.

Lemma 4.2. If $\mu$ is centered Gaussian with covariance operator $A$, then Spt $\mu=A\left(R^{d}\right)$.

Proof of Theorem 4.2. Let $\hat{\mu}(z)=\exp \left(2^{-1} \phi_{A}(z)\right)$. By (4.7) of Theorem 4.1, we have 


$$
U_{k} A U_{j}^{\prime}=0 \quad \text { for } j \neq k \text {. }
$$

It follows that

$$
A=\sum_{j=1}^{q+r} U_{j} A U_{j}^{\prime}
$$

Let $A_{j}=U_{j} A U_{j}^{\prime}$. Note that, by (4.12), $A_{j}=U_{j} A=A U_{j}^{\prime}$. Let $\mu_{j}$ be centered Gaussian with covariance operator $A_{j}$. Then we have (4.11) and $A_{j}\left(R^{d}\right) \subset W_{j}$. Hence Spt $\mu_{j} \subset W_{j}$ by Lemma 4.2. The uniqueness of the decomposition is obvious. Using Theorem 3.1 and the relations between $\left\{U_{j}\right\}$ and $\left\{T_{j}\right\}$, we see that $\mu_{j} \in L_{\infty}(Q)$ and Spt $\mu_{j} \subset N_{j}$. If $1 \leq j \leq q$, then, for each $x \in R^{d}, Q A_{j} x=Q A T_{j}^{\prime} x=\alpha_{j} A T_{j}^{\prime} x \in A_{j}\left(R^{d}\right)$ by (3.5). If $q+1 \leq$ $j \leq q+r$, then, for each $x \in R^{d}, Q A_{j} x=Q A\left(T_{j}^{\prime}+T_{j+r}^{\prime}\right) x=\alpha_{j} A T_{j}^{\prime} x+$ $\bar{\alpha}_{j} A T_{j+r}^{\prime} x=2 \operatorname{Re}\left(\alpha_{j} A T_{j}^{\prime} x\right)=\beta_{j} A U_{j}^{\prime} x-\gamma_{j} A y_{j} \in A_{j}\left(R^{d}\right)$, where $y_{j}=2 \operatorname{Im} T_{j}^{\prime} x$ $=i^{-1}\left(T_{j}^{\prime}-T_{j+r}^{\prime}\right) x \in W_{j}^{\prime}$. Hence, $A_{j}\left(R^{d}\right)$ is $Q$-invariant. Hence, $A\left(R^{d}\right)$ is $Q$-invariant. Since it is contained in $N_{1} \oplus \cdots \oplus N_{q+r}$, the restriction of $Q$ to it has a minimal polynomial without double roots. The proof is complete. (Another proof of $Q$-invariance of $\operatorname{Spt} \mu$ is to use Proposition 4.1 of Yamazato [21]. Indeed, it is true for all centered Gaussian distributions $\mu$ in $L_{0}(Q)$.)

Remark 4.1. Assume that all eigenvalues of $Q$ are real. Then the following converse of Theorem 4.2 is true: If $\mu_{1}, \cdots, \mu_{q}$ are centered Gaussian distributions such that Spt $\mu_{j} \subset N_{j}$ for each $j$, then the convolution $\mu=\mu_{1} * \cdots * \mu_{q}$ belongs to $L_{\infty}(Q)$. In fact, $\mu_{j} \in L_{\infty}(Q)$ as a consequence of Theorem 3.1 and Lemma 4.2, and the class $L_{\infty}(Q)$ is closed under convolution.

\section{$\S 5$. Purely non-Gaussian completely operator-selfdecomposable distributions}

Let us describe conditions for centered purely non-Gaussian distributions to belong to $L_{\infty}(Q)$. We continue to use the notations in the preceding sections on the decompositions of $R^{d}$ and $C^{d}$ induced by $Q \in$ $M_{+}\left(R^{d}\right)$. For $\xi \in R^{d}$ with $|\xi|=1$, we define $\beta_{\xi}=\beta(\xi)$ and $n_{\xi}=n(\xi)$ as follows:

$$
\begin{aligned}
& \beta_{\xi}=\min \left\{\beta_{j}: 1 \leq j \leq q+r, U_{j} \xi \neq 0\right\}=\min \left\{\beta_{j}: 1 \leq j \leq q+2 r, T_{j} \xi \neq 0\right\}, \\
& n(\xi, j)=\max \left\{n: n \geq 0,\left(Q-\alpha_{j}\right)^{n} T_{j} \xi \neq 0\right\} \quad \text { for } T_{j} \xi \neq 0, \\
& n_{\xi}=\max \left\{n(\xi, j): 1 \leq j \leq q+2 r, T_{j} \xi \neq 0, \beta_{j}=\beta_{\xi}\right\} .
\end{aligned}
$$


Note that, if $q+1 \leq j \leq q+r$ and $x \in R^{d}$, then $U_{j} x=0$ and $T_{j} x=0$ are equivalent. A system of measures $\left\{\Gamma_{\xi}\right\}$ is said to be measurable in $\xi$ if $\Gamma_{\xi}(E)$ is measurable in $\xi$ for each Borel set $E$. The following is a theorem similar to results of Sato [7] and Jurek [3].

THEOREM 5.1. If $\mu$ is a centered, purely non-Gaussian distribution in $L_{\infty}(Q)$, then

$$
\hat{\mu}(z)=\exp \int_{S_{Q}} \lambda(d \xi) \int_{(0,2 \beta \xi)} \Gamma_{\xi}(d \alpha) \int_{0}^{\infty} g\left(z, u^{Q \xi}\right) u^{-\alpha-1} d u,
$$

where $\lambda$ is the zero measure or a probability measure on $S_{Q}$ and $\Gamma_{\xi}(d \alpha)$ is a measure on $\left(0,2 \beta_{\xi}\right)$ such that the system $\left\{\Gamma_{\xi}\right\}$ is measurable in $\xi$ and

$$
0<\int_{(0,2 \beta \xi)} \Gamma_{\xi}(d \alpha) \int_{0}^{\infty} h\left(\left|u^{Q \xi}\right|\right) u^{-\alpha-1} d u=c<\infty
$$

with $c$ independent of $\xi$. This $\lambda$ is uniquely determined by $\mu$ and this $\Gamma_{\xi}(d \alpha)$ is uniquely determined by $\mu$ up to $\xi$ of a set of $\lambda$-measure zero. Conversely, given any $\lambda$ and $\Gamma_{\xi}(d \alpha)$ with the above properties, one can find $\mu \in L_{\infty}(Q)$ described by (5.1). If a measure $\Gamma_{\xi}(d \alpha)$ on $\left(0,2 \beta_{\xi}\right)$ satisfies (5.2), then

$$
0<\int_{(0,2 \beta \xi)}\left(\alpha^{-1}+\left(2 \beta_{\xi}-\alpha\right)^{-n(\xi)-1}\right) \Gamma_{\xi}(d \alpha)<\infty .
$$

If a family of measures $\left\{\Gamma_{\xi}^{0}(d \alpha)\right\}$ satisfies (5.3) for each $\xi$ with $\Gamma_{\xi}$ replaced by $\Gamma_{\xi}^{0}$, and if $\left\{\Gamma_{\xi}^{0}\right\}$ is measurable in $\xi$, then one can find a positive measurable function $a(\xi)$ such that $\Gamma_{\xi}(d \alpha)=a(\xi) \Gamma_{\xi}^{0}(d \alpha)$ satisfies (5.2) with $c$ independent of $\xi$.

For $Q \in M_{+}\left(R^{d}\right)$, we denote by $c_{k}$ positive constants that depend only on $Q$. By $b_{k}(\xi)$ we denote positive functions that depend only on $Q$.

Lemma 5.1. There are $c_{k}(k=5,6,7)$ and $b_{k}(\xi)(k=1,2,3)$ such that, for $|\xi|=1$,

$$
\begin{gathered}
\left|u^{Q} \xi\right| \leq c_{5} u^{\beta(\xi)}|\log u|^{n(\xi)} \quad \text { fot } 0<u<1 / 3, \\
\left|u^{Q} \xi\right| \geq b_{2}(\xi) u^{\beta(\xi)}|\log u|^{n(\xi)} \quad \text { for } 0<u<b_{1}(\xi), \\
c_{6} \alpha^{-1}+b_{3}(\xi)\left(2 \beta_{\xi}-\alpha\right)^{-2 n(\xi)-1} \leq \int_{0}^{\infty} h\left(\left|u^{Q} \xi\right|\right) u^{-\alpha-1} d u \\
\leq c_{7}\left(\alpha^{-1}+\left(2 \beta_{\xi}-\alpha\right)^{-2 n(\xi)-1}\right) \quad \text { for } 0<\alpha<2 \beta_{\xi} .
\end{gathered}
$$

If $\alpha \geq 2 \beta_{\xi}$, then, for $|\xi|=1$, 


$$
\int_{0}^{\infty} h\left(\left|u^{Q} \xi\right|\right) u^{-\alpha-1} d u=\infty
$$

Proof. We have

$$
u^{Q} T_{j} \xi=u^{\alpha(j)} \sum_{n=0}^{n(\xi, j)}(n !)^{-1}(\log u)^{n}\left(Q-\alpha_{j}\right)^{n} T_{j} \xi,
$$

where $\alpha(j)=\alpha_{j}$. Hence

$$
\left|u^{Q} \xi\right| \leq c_{8} \sum_{j=1}^{p} u^{\beta(j)}|\log u|^{n(\xi, j)} \quad \text { for } 0<u<1 / 3,
$$

where $\beta(j)=\beta_{j}$. Hence (5.4). It follows from (5.8) that there are $b_{1}(\xi)$ and $b_{4}(\xi)$ such that, for $0<u<b_{1}(\xi)$,

$$
\begin{aligned}
\left|u^{Q} T_{j} \xi\right| & \geq 2^{-1} u^{\beta(j)}(n(\xi, j) !)^{-1}|\log u|^{n(\xi, j)}\left|\left(Q-\alpha_{j}\right)^{n(\xi, j)} T_{j} \xi\right| \\
& \geq b_{4}(\xi) u^{\beta(j)}|\log u|^{n(\xi, j)}
\end{aligned}
$$

for all $j$ satisfying $T_{j} \xi \neq 0$. Choose a norm $\|\cdot\|$ in $C^{a}$ such that $\|x\|=$ $\sum_{j=1}^{p}\left\|T_{j} x\right\|$. Since arbitrary two norms are equivalent, we have $a_{1}|x| \leq$ $\|x\| \leq a_{2}|x|$ for some positive $a_{1}$ and $a_{2}$. Choosing $j$ such that $\beta_{\xi}=\beta_{j}$ and $n_{\xi}=n(\xi, j)$, we obtain

$$
\left|u^{Q} \xi\right| \geq a_{2}^{-1}\left\|u^{Q} \xi\right\| \geq a_{2}^{-1}\left\|u^{Q} T_{j} \xi\right\| \geq a_{1} a_{2}^{-1}\left|u^{Q} T_{j} \xi\right| \text {. }
$$

Hence (5.5) follows. Let $0<\alpha<2 \beta_{\xi}$. We have

$$
\begin{aligned}
& \int_{0}^{1 / 3} h\left(\left|u^{Q \xi}\right|\right) u^{-\alpha-1} d u \leq \int_{0}^{1 / 3}\left|u^{Q} \xi\right|^{2} u^{-\alpha-1} d u \\
& \quad \leq c_{5}^{2} \int_{0}^{1 / 3} u^{2 \beta(\xi)-\alpha-1}|\log u|^{2 n(\xi)} d u \leq c_{5}^{2} \Gamma\left(2 n_{\xi}+1\right)\left(2 \beta_{\xi}-\alpha\right)^{-2 n(\xi)-1}
\end{aligned}
$$

from (5.4), and

$$
\int_{1 / 3}^{\infty} h\left(\left|u^{Q} \xi\right|\right) u^{-\alpha-1} d u \leq \int_{1 / 3}^{\infty} u^{-\alpha-1} d u \leq c_{9} \alpha^{-1} .
$$

This proves the second inequality in (5.6). The first inequality is obtained from (5.5) as follows. We may suppose $b_{1}(\xi)<1$. Since $h(s)$ is increasing in $s>0$, we have

$$
\begin{aligned}
\int_{0}^{b_{1}(\xi)} h\left(\left|u^{Q} \xi\right|\right) u^{-\alpha-1} d u \geq \int_{0}^{b_{1}(\xi)} h\left(b_{2}(\xi) u^{\beta(\xi)}|\log u|^{n(\xi)}\right) u^{-\alpha-1} d u \\
\quad \geq b_{5}(\xi) \int_{0}^{b_{1}(\xi)} u^{2 \beta(\xi)-\alpha-1}|\log u|^{2 n(\xi)} d u \\
\quad=b_{5}(\xi)(2 \beta(\xi)-\alpha)^{-2 n(\xi)-1} \int_{(2 \beta(\xi)-\alpha)\left|\log b_{1}(\xi)\right|}^{\infty} e^{-s} s^{2 n(\xi)} d s \\
\geq b_{6}(\xi)(2 \beta(\xi)-\alpha)^{-2 n(\xi)-1} .
\end{aligned}
$$


Since $h\left(\left|u^{Q} \xi\right|\right) \geq c_{6}$ for $u \geq 1$, we have

$$
\int_{1}^{\infty} h\left(\left|u^{Q} \xi\right|\right) u^{-\alpha-1} d u \geq c_{6} \alpha^{-1}
$$

Hence the first inequality in (5.6). The proof of (5.7) for $\alpha \geq 2 \beta_{\xi}$ is similar.

Proof of Theorem 5.1. For $\mu \in L_{\infty}(Q)$, the function $k_{\xi}(u)$ in the first representation is such that $k_{\xi}\left(e^{t}\right)$ is completely monotone in $-\infty<t<\infty$ for $\lambda$-almost every $\xi$ (Theorem 2.1). By modification we may assume the exceptional set of $\xi$ is void. By Bernstein's theorem, there is, for each $\xi$, a unique finite measure $\Gamma_{\xi}(d \alpha)$ on $[0, \infty)$ such that

$$
k_{\xi}\left(e^{t}\right)=\int_{[0, \infty)} e^{-\alpha t} \Gamma_{\xi}(d \alpha) \text {. }
$$

The system $\left\{\Gamma_{\xi}\right\}$ is measurable in $\xi$. Since $k_{\xi}(u) \rightarrow 0$ as $u \rightarrow \infty$ by (2.6), we have $\Gamma_{\xi}(\{0\})=0$. Rewriting (2.6), we get (5.2) with the domain of integration $\left(0,2 \beta_{\xi}\right)$ replaced by $(0, \infty)$. By $(5.7)$ of Lemma 5.1 , this shows that $\Gamma_{\xi}(d \alpha)$ is concentrated on $\left(0,2 \beta_{\xi}\right)$ and we have the representation (5.1). The uniqueness assertion is obvious from the uniqueness of the representation (2.4). The converse assertion follows from that of Theorem 2.1. The assertion (5.3) follows from (5.2) by (5.6) of Lemma 5.1. If a family $\left\{\Gamma_{\xi}^{0}\right\}$ satisfies (5.3), then, let

$$
a(\xi)=\left(\int_{(0,2 \beta \xi)} \Gamma_{\xi}^{0}(d \alpha) \int_{0}^{\infty} h\left(\left|u^{Q} \xi\right|\right) u^{-\alpha-1} d u\right)^{-1} .
$$

This is a positive function by (5.6). The measure $\Gamma_{\xi}(d \alpha)=a(\xi) \Gamma_{\xi}^{0}(d \alpha)$ satisfies (5.2) with $c=1$. The proof is complete.

Let us give some results on supports of operator-selfdecomposable purely non-Gaussian distributions. A measure $\mu$ in $R^{d}$ is said to be genuinely $d$-dimensional (or full), if no $(d-1)$-dimensional hyperplane contains Spt $\mu$. A measure $\mu$ is said to be full in an $\ell$-dimensional affine subspace $W$ of $R^{d}$, if Spt $\mu \subset W$ and $\mu$ is genuinely $\ell$-dimensional. The following fact is used in Sato [8] and Yamazato [20].

LEMMA 5.2. Let $\mu$ be a centered, purely non-Gaussian distribution in $R^{a}$ with Lévy measure $\nu \neq 0$. Denote by $W_{\mu}$ or $W_{\nu}$ the smallest linear subspace that contains Spt $\mu$ or Spt $\nu$, respectively. Then $W_{\mu}=W_{\nu}$ and $\mu$ is full in $W_{\mu}$.

Proof. We have Spt $\mu \subset W_{\nu}$ since $\hat{\mu}(z)=1$ for $z$ in the orthogonal 
complement $W_{\nu}^{\perp}$ of $W_{\nu}$. Hence $W_{\mu} \subset W_{\nu}$. Let $\mu_{0}$ be the symmetrization of $\mu$. Then Spt $\mu_{0} \subset W_{\mu}$. Let $z_{0}$ be orthogonal to $\operatorname{Spt} \mu_{0}$. Then

$$
1=\hat{\mu}_{0}\left(t z_{0}\right)=\exp \left(2 \int\left(\cos \left\langle x, t z_{0}\right\rangle-1\right) \nu(d x)\right),
$$

and hence $\cos \left\langle x, t z_{0}\right\rangle=1$ for $\nu$-almost every $x$. Hence $\left\langle x, z_{0}\right\rangle=0$ for $\nu$-almost every $x$, that is, $W_{\nu} \subset\left\{z_{0}\right\}^{\perp}$. It follows that $W_{\mu}=W_{\nu}$. If $\mu$ is not full in $W_{\mu}$, then Spt $\mu_{0}$ is contained in a proper subspace of $W_{\mu}$ and, by the argument above, there is a non-zero $z_{0}$ in $W_{\mu}$ such that $W_{\nu} \subset\left\{z_{0}\right\}^{\perp}$, which is absurd. Therefore $\mu$ is full in $W_{\mu}$.

TheOREM 5.2. If $\mu$ is a centered, purely non-Gaussian distribution in $L_{0}(Q)$, then $\mu$ is full in a Q-invariant linear subspace of $R^{d}$.

Proof. Let $\nu$ be the Lévy measure of $\mu$. If $\nu=0$, then the assertion is trivial. Suppose $\nu \neq 0$. Let $\lambda$ be the probability measure in the first representation of $\mu$. Let $W$ be the smallest $Q$-invariant linear subspace that contains Spt $\lambda$. Then, by Lemma 5 of Yamazato [20], $W$ is the smallest linear subspace that contains Spt $\nu$. Hence, by Lemma 5.2, $\mu$ is full in $W$.

\section{§6. Operator-stable distributions}

Let $Q \in M_{+}\left(R^{d}\right)$ and $\alpha>0$. We call a distribution $\mu$ operator-stable with exponent $(\alpha, Q)$ or, in short, $(\alpha, Q)$-stable, if $\mu \in I D\left(R^{d}\right)$ and, for every $t>0$, there is $a(t) \in R^{d}$ such that

$$
\mu^{t^{\alpha}}=t^{Q} \mu * \delta_{a(t)} .
$$

The relation (6.1) is equivalent to

$$
\mu^{t}=t^{\alpha-1} \mu * \delta_{b(t)}
$$

with some $b(t) \in R^{d}$. Thus $\mu$ is operator-stable with exponent $(\alpha, Q)$ if and only if $\mu$ is operator-stable with exponent $\left(1, \alpha^{-1} Q\right)$. In Sharpe's terminology in [12], it is operator-stable with exponent $\alpha^{-1} Q$. In our new naming, stable distributions with exponent $\alpha$ are $(\alpha, I)$-stable, and vice versa. Results on stable distributions with exponent $\alpha$ thus find natural generalization to $(\alpha, Q)$-stable distributions. Sharpe [12] determines conditions on $Q$ in order that there exist full $(1, Q)$-stable distributions. Further he finds a structure of general full operator-stable distributions and an expression of their Lévy measures (see also Hudson-Mason [1]). 
But he does not determine the whole structure of Gaussian operatorstable distributions. It is done by Schmidt [11] in some degree. We will describe all operator-stable distributions without the fullness assumption. The following two lemmas are basic.

LEMMA 6.1 (Jurek [2]). If $\mu$ is $(\alpha, Q)$-stable for some $\alpha>0$, then $\mu \in$ $L_{\infty}(Q)$.

Proof. For $0<t<1$, we have

$$
\mu=\mu^{t \alpha} * \mu^{1-t^{\alpha}}=t^{Q} \mu * \delta_{a(t)} * \mu^{1-t^{\alpha}} .
$$

Hence $\mu \in L_{0}(Q)$. It follows that $\mu_{t}=\delta_{a(t)} * \mu^{1-t^{\alpha}} \in L_{0}(Q)$. Hence $\mu \in L_{1}(Q)$ by Proposition 1.1. Repeating this, we get $\mu \in L_{\infty}(Q)$.

LEMMA 6.2 (Sharpe [12]). Let $\mu$ be infinitely divisible with Lévy representation $(\gamma, A, \nu)$. Then, $\mu$ is $(\alpha, Q)$-stable if and only if

$$
\phi_{A}\left(t^{Q^{\prime}} z\right)=t^{\alpha} \phi_{A}(z) \quad \text { for } z \in R^{d}, \quad t>0,
$$

and

$$
\left(t^{Q} \nu\right)(E)=t^{\alpha} \nu(E) \quad \text { for } E \in \mathscr{B}\left(R^{a}\right), \quad t>0 .
$$

Proof. By (6.2) it is enough to consider only the case $\alpha=1$. If $\alpha=1$, this is Proposition 5 of Sharpe [12].

We continue to use the decompositions (3.2), (3.4), (4.3), and (4.9) associated with $Q$ and the projectors $T_{j}, T_{j}^{\prime}, U_{j}$, and $U_{j}^{\prime}$.

Theorem 6.1. Let $\mu$ be Gaussian with covariance operator A. Then, $\mu$ is $(\alpha, Q)$-stable if and only if (3.5) is satisfied and

$$
A T_{j}^{\prime}=0 \quad \text { for every } j \text { such that } \operatorname{Re} \alpha_{j} \neq \alpha / 2 \text {. }
$$

Proof. Suppose that $\mu$ is $(\alpha, Q)$-stable. By Theorem 3.1 and Lemma 6.1, it satisfies (3.5). Since (6.3) holds also for $z \in C^{d}$, we have, noting $e^{-t Q^{\prime}} T_{j}^{\prime} z \in V_{j}^{\prime}$ and using (3.5),

$$
e^{-t \alpha} \phi_{A}\left(T_{j}^{\prime} z\right)=\phi_{A}\left(e^{-t Q^{\prime}} T_{j}^{\prime} z\right)=e^{-2 t \operatorname{Re} \alpha(j)} \phi_{A}\left(T_{j}^{\prime} z\right),
$$

where $\alpha(j)=\alpha_{j}$. Hence we obtain (6.5). Conversely, if (3.5) and (6.5) are satisfied, then we get, using (3.7),

$$
\phi_{A}\left(e^{-t Q^{\prime}} z\right)=\sum_{j=1}^{p} \phi_{A}\left(e^{-t Q^{\prime}} T_{j}^{\prime} z\right)=\sum_{j=1}^{p} e^{-2 t \operatorname{Re} \alpha(j)} \phi_{A}\left(T_{j}^{\prime} z\right)=e^{-\alpha t} \phi_{A}(z),
$$

which implies $(\alpha, Q)$-stability of $\mu$ by Lemma 6.2. The proof is complete. 
THEOREM 6.2. Let $\mu$ be a purely non-Gaussian infinitely divisible distribution with Lévy measure $\nu$. Let $W_{(\alpha)}$ be the direct sum of all the subspaces $W_{j}$ for which $\operatorname{Re} \alpha_{j}>\alpha / 2$. Then $\mu$ is $(\alpha, Q)$-stable if and only if

$$
\nu(E)=\int_{S_{Q} \cap W_{(\alpha)}} \lambda_{0}(d \xi) \int_{0}^{\infty} \chi_{E}\left(u^{\natural} \xi\right) u^{-\alpha-1} d u \quad \text { for } E \in \mathscr{B}\left(R^{a}\right),
$$

where $\lambda_{0}$ is a finite measure on $S_{Q} \cap W_{(\alpha)}$. For every finite measure $\lambda_{0}$ on $S_{Q} \cap W_{(\alpha)}$, there exists an $(\alpha, Q)$-stable purely non-Gaussian distribution with Lévy measure $\nu$ described by (6.6).

Proof. Assume that $\mu$ is $(\alpha, Q)$-stable. For any $F \in \mathscr{B}\left(S_{Q}\right)$, let $\tilde{F}=$ $\left\{u^{q} \xi: \xi \in F, u>1\right\}$ and define $\lambda_{0}(F)=\alpha \nu(\tilde{F})$. Then $\lambda_{0}$ is a finite measure on $S_{Q}$, since $\tilde{F} \subset\{x:|x|>1\}$. Define a measure $\nu_{1}$ by

$$
\nu_{1}(E)=\int_{S_{Q}} \lambda_{0}(d \xi) \int_{0}^{\infty} \chi_{E}\left(u^{Q} \xi\right) u^{-\alpha-1} d u \quad \text { for } E \in \mathscr{B}\left(R^{d}\right) .
$$

Let $a>0$ and $E=\left\{u^{Q} \xi: \xi \in F, u>a\right\}$. Then

$$
\nu_{1}(E)=\int_{F} \lambda_{0}(d \xi) \int_{a}^{\infty} u^{-\alpha-1} d u=\alpha^{-1} a^{-\alpha} \lambda_{0}(F)=a^{-\alpha} \nu(\tilde{F}) .
$$

Since $\tilde{F}=\left\{(u / a)^{Q} \xi: \xi \in F, u>a\right\}=(1 / a)^{Q} E$, we have

$$
a^{-\alpha} \nu(\tilde{F})=a^{-\alpha}\left(a^{\alpha} \nu\right)(E)=\nu(E),
$$

using (6.4) of Lemma 6.2. Hence $\nu_{1}(E)=\nu(E)$. It follows that $\nu_{1}=\nu$. We have

$$
\begin{aligned}
\infty>\int h(|x|) \nu(d x)= & \int_{S_{Q}} \lambda_{0}(d \xi) \int_{0}^{\infty} h\left(\left|u^{Q} \xi\right|\right) u^{-\alpha-1} d u \\
& \geq \int_{S_{Q}} \lambda_{0}(d \xi) \int_{0}^{b_{1}(\xi)} h\left(b_{2}(\xi) u^{\beta(\xi)}\right) u^{-\alpha-1} d u,
\end{aligned}
$$

using (5.5) of Lemma 5.1 and choosing $b_{1}(\xi) \leq 1 / 3$. Since

$$
\int_{0}^{b_{1}(\xi)} h\left(b_{2}(\xi) u^{\beta(\xi)}\right) u^{-\alpha-1} d u=\infty \quad \text { for } \beta(\xi) \leq \alpha / 2,
$$

we see that $\beta(\xi)>\alpha / 2$ for $\lambda_{0}$-almost every $\xi$, that is, $\lambda_{0}$ is concentrated in $S_{Q} \cap W_{(\alpha)}$. Conversely, if the Lévy measure $\nu$ of $\mu$ is expressed as (6.6) with some $\lambda_{0}$, then (6.4) is an easy consequence and $\mu$ is $(\alpha, Q)$-stable. In order to prove the last sentence in the theorem, it is enough to show that, for any finite measure $\lambda_{0}$ on $S_{Q} \cap W_{(\alpha)}$, the measure $\nu$ defined by (6.6) satisfies $\int h(|x|) \nu(d x)<\infty$. Using (5.6) of Lemma 5.1, we get 


$$
\int \lambda_{0}(d \xi) \int_{0}^{\infty} h\left(\left|u^{Q \xi}\right|\right) u^{-\alpha-1} d u \leq c_{7} \int \lambda_{0}(d \xi)\left(\alpha^{-1}+\left(2 \beta_{\xi}-\alpha\right)^{-2 n(\xi)-1}\right),
$$

which is finite, since, for some $\varepsilon>0, \beta(\xi)>2^{-1} \alpha+\varepsilon$ uniformly in $\xi \in W_{(\omega)}$ and since $n(\xi) \leq d$. The proof is complete.

Remark 6.1. A purely non-Gaussian distribution $\mu$ is $(\alpha, Q)$-stable if and only if $\mu \in L_{0}(Q)$ and, in the first representation of $\mu$,

$$
k_{\xi}(u)=a(\xi) u^{-\alpha},
$$

$a(\xi)$ is measurable and $0<a_{1} \leq a(\xi) \leq a_{2}<\infty$

with some constants $a_{1}, a_{2}$, and

$$
\lambda(d \xi) \text { is concentrated in } S_{Q} \cap W_{(\alpha)} .
$$

In fact, it is obvious that (6.7)-(6.9) imply (6.6). Choose $\varepsilon>0$ so that there is no eigenvalue $\alpha_{j}$ satisfying $2^{-1} \alpha<\operatorname{Re} \alpha_{j} \leq 2^{-1} \alpha+\varepsilon$. There are constants $c_{2}, c_{3}$, and $c_{4}$ such that (2.1) and (2.2) hold with $c_{1}=2^{-1} \alpha+\varepsilon$ for all $x \in W_{(\alpha)}$ (Urbanik [15] p. 139). It follows that there are positive constants $c_{5}$ and $c_{6}$ such that

$$
c_{5} \leq \int_{0}^{\infty} h\left(\left|u^{Q} \xi\right|\right) u^{-\alpha-1} d u \leq c_{6}
$$

for all $\xi \in S_{Q} \cap W_{(\alpha)}$. If $\mu$ is $(\alpha, Q)$-stable, we choose

$$
\begin{array}{cc}
b(\xi)=\left(\int_{0}^{\infty} h\left(\left|u^{Q} \xi\right|\right) u^{-\alpha-1} d u\right)^{-1}, & a(\xi)=b(\xi) \int_{S_{Q} \cap W_{(\alpha)}} b(\eta)^{-1} \lambda_{0}(d \eta), \\
k_{\xi}(u)=a(\xi) u^{-\alpha}, & \lambda(d \xi)=a(\xi)^{-1} \lambda_{0}(d \xi),
\end{array}
$$

which are shown to give the first representation satisfying (6.7)-(6.9).

An arbitrary $(\alpha, Q)$-stable distribution is decomposed into two $(\alpha, Q)$ stable distributions, one of which is Gaussian and the other is purely non-Gaussian, together with a decomposition of the basic space $R^{d}$. This is shown by the following theorem. Note that we do not impose the fullness assumption.

THEOREM 6.3. Let $\mu \in I D\left(R^{a}\right)$, let $\mu_{1}$ be the centered Gaussian component of $\mu$ and let $\mu_{2}$ be the centered purely non-Gaussian component of $\mu$. Then, $\mu$ is $(\alpha, Q)$-stable if and only if there exist Q-invariant linear subspaces $W^{(1)}$ and $W^{(2)}$ such that $W^{(1)} \cap W^{(2)}=\{0\}$ and, for each $j, \mu_{j}$ is full in $W^{(j)}$ and $\left(\alpha, Q_{j}\right)$-stable as a measure on $W^{(j)}$, where $Q_{j}$ is the restriction of $Q$ to $W^{(j)}$. 
Remark 6.2. The theorem above reduces general $(\alpha, Q)$-stable distributions to the full case studied by Sharpe [12]. Thus the eigenvalues of the operator $Q_{1}$ have real parts equal to $\alpha / 2$ and the minimal polynomial of $Q_{1}$ does not have double roots, while the eigenvalues of $Q_{2}$ have real parts greater than $\alpha / 2$. But note that there does not necessarily exist a $Q$-invariant linear subspace $W^{(3)}$ such that $R^{d}=W^{(1)} \oplus W^{(2)} \oplus W^{(3)}$.

Proof of Theorem 6.3. Suppose that $\mu$ is $(\alpha, Q)$-stable. Then, by Lemma 6.2 , both $\mu_{1}$ and $\mu_{2}$ are $(\alpha, Q)$-stable. Let $W^{(1)}$ be Spt $\mu_{1}$, and let $W^{(2)}$ be the smallest linear subspace containing Spt $\mu_{2}$. Recalling Ləmma 6.1, we can freely use our theorems on $L_{\infty}(Q)$. By Theorem $4.2, W^{(1)}$ is $Q$-invariant. By Theorem 5.2, $W^{(2)}$ is $Q$-invariant and $\mu_{2}$ is full in $W^{(2)}$. Denote by $\left[\mu_{j}\right]_{(j)}$ the restriction of $\mu_{j}$ to $W^{(j)}$. We see that $\left[\mu_{j}\right]_{(j)}$ is $\left(\alpha, Q_{j}\right)$-stable. For we have

$$
t^{Q j}\left[\mu_{j}\right]_{(j)}=\left[t^{Q} \mu_{j}\right]_{(j)}=\left[\mu_{j}^{t \alpha} * \delta_{-a_{j}(t)}\right]_{(j)}=\left(\left[\mu_{j}\right]_{(j)}\right)^{t \alpha} * \delta_{-a_{j}(t)},
$$

where $a_{j}(t) \in W^{(j)}$. By Theorem 6.1 and Lemma 4.2, the eigenvalues of $Q_{1}$ have real parts $\alpha / 2$, while, by Theorem 6.2 and Lemma 5.2, the real parts of the eigenvalues of $Q_{2}$ are greater than $\alpha / 2$. Hence we have $W^{(1)} \cap W^{(2)}$ $=\{0\}$.

Conversely, suppose that $W^{(1)}$ and $W^{(2)}$ are $Q$-invariant linear subspaces and that, for $j=1,2, \mu_{j}$ is a probability measure with support in $W^{(j)}$ and $\left[\mu_{j}\right]_{(j)}$ is $\left(\alpha, Q_{j}\right)$-stable. Then it is easy to see that $\mu_{j}$ is $(\alpha, Q)$ stable and that $\mu_{1} * \mu_{2}$ is also $(\alpha, Q)$-stable. The proof is complete.

\section{§7. Relations between completely operator-selfdecomposable distri- butions and operator-stable distributions}

We will establish relations between the classes $L_{\infty}(Q)$ and $S(Q)$, which extend results of Urbanik [16], [17], Thu [13], and Sato [7]. By Proposition 1.2 , the class $S(Q)$ is the totality of probability distributions which are $(\alpha, Q)$-stable for some $\alpha>0$.

TheOREM 7.1. Let $s$ be the number of distinct real parts of eigenvalues of $Q$. If $\mu$ is a Gaussian distribution in $L_{\infty}(Q)$, then $\mu$ can be expressed as the convolution of at most $s$ Gaussian distributions in $S(Q)$.

Proof. We may suppose that $\mu$ is centered. If $\mu \in L_{\infty}(Q)$, then, by Theorem 6.1, the decomposition of $\mu$ in Theorem 4.2 gives representation of $\mu$ as a convolution of Gaussian distributions that belong to $S(Q)$. Again 
by Theorem 6.1 we see that it is written as the convolution of at most $s$ Gaussians in $S(Q)$.

TheOREm 7.2. If $\mu \in L_{\infty}(Q)$, then $\mu$ is the limit of a sequence $\left\{\mu_{n}\right\}$ such that each $\mu_{n}$ is a convolution of a finite number of purely non-Gaussian distributions in $S(Q)$.

Proof. Let $\mu \in L_{\infty}(Q)$. We may assume that it is centered. Consider the case where $\mu$ is purely non-Gaussian with the expression (5.1) such that, for some $\varepsilon>0, \Gamma_{\xi}(d \alpha)$ is concentrated in $\left(\varepsilon, 2 \beta_{\xi}-\varepsilon\right)$ for each $\xi \in S_{Q}$. Then we can choose measures $\Gamma_{\xi}^{(n)}(d \alpha)$ satisfying the following conditions: $\Gamma_{\xi}^{(n)}(d \alpha)$ is concentrated on the points $\left\{2^{-n} k: k=1,2, \cdots\right\}$ in the interval $\left(\varepsilon, 2 \beta_{\xi}-\varepsilon\right), \Gamma_{\xi}^{(n)}(d \alpha)$ converges to $\Gamma_{\xi}(d \alpha)$ for each $\xi$ as $n \rightarrow \infty$, the total mass of $\Gamma_{\xi}^{(n)}(d \alpha)$ does not exceed that of $\Gamma_{\xi}(d \alpha)$ for each $\xi$, and $\left\{\Gamma_{\xi}^{(n)}\right\}$ is measurable in $\xi$. Define $\mu_{n}$ by

$$
\hat{\mu}_{n}(z)=\exp \int_{S_{Q}} \lambda(d \xi) \int_{(\varepsilon, 2 \beta \xi-\varepsilon)} \Gamma_{\xi}^{(n)}(d \alpha) \int_{0}^{\infty} g\left(z, u^{Q} \xi\right) u^{-\alpha-1} d u .
$$

By Lemma 5.1 and by $|g(z, x)| \leq c_{z} h(|x|)$, the integral $\int_{0}^{\infty} g\left(z, u^{\ell} \xi\right) u^{-\alpha-1} d u$ is bounded in $\alpha \in\left(\varepsilon, 2 \beta_{\xi}-\varepsilon\right)$ and $\xi \in S_{Q}$ and is continuous in $\alpha$. Hence

$$
\int \Gamma_{\xi}^{(n)}(d \alpha) \int g\left(z, u^{Q} \xi\right) u^{-\alpha-1} d u \rightarrow \int \Gamma_{\xi}(d \alpha) \int g\left(z, u^{Q} \xi\right) u^{-\alpha-1} d u
$$

as $n \rightarrow \infty$. Since (5.2) and (5.6) imply that the total mass of $\Gamma_{\xi}(d \alpha)$ is bounded in $\xi \in S_{Q}$, we see that $\hat{\mu}_{n}(z) \rightarrow \hat{\mu}(z)$. It follows from Theorem 6.2 that $\mu_{n}$ is a convolution of a finite number of purely non-Gaussian distributions in $S(Q)$. If $\mu$ is purely non-Gaussian, then the assertion is proved by approximation of its $\Gamma_{\xi}(d \alpha)$ measure by $\chi_{(\varepsilon, 2 \beta \xi-\varepsilon)}(\alpha) \Gamma_{\varepsilon}(d \alpha)$ as $\varepsilon \downarrow 0$. Now recall Theorem 7.1. Then we see that, in order to complete the proof, it is enough to show that any $(\alpha, Q)$-stable Gaussian distribution $\mu$ is a limit of purely non-Gaussian distributions in $S(Q)$. Let $A$ be the covariance operator of $\mu$. For $0<\beta<1$ let $\mu_{\beta}^{0}$ be a symmetric, $(2 \beta, I)$-stable distribution such that $\hat{\mu}_{\beta}^{0}(z)=\exp \left(-2^{-1}|z|^{2 \beta}\right)$. Let $\mu_{\beta}=A^{1 / 2} \mu_{\beta}^{0}$. Since $\mu_{\beta}^{0}$ is purely non-Gaussian, so is $\mu_{\beta}$. Since

$$
\hat{\mu}_{\beta}(z)=\exp \left(-2^{-1} \phi_{A}(z)^{\beta}\right),
$$

we have, by (6.3),

$$
\hat{\mu}_{\beta}\left(t^{Q^{\prime}} z\right)=\hat{\mu}_{\beta}(z)^{t^{\alpha \beta}}
$$


Hence $\mu_{\beta}$ is $(\alpha \beta, Q)$-stable. As $\beta \uparrow 1, \mu_{\beta}$ tends to $\mu$. The proof is complete. As a consequence we have the following.

TheOREM 7.3. The class $L_{\infty}(Q)$ is the smallest class closed under convolution and convergence and containing the class $S(Q)$.

\section{REFERENCES}

[1] Hudson, W. N. and Mason, J. D., Operator-stable laws, J. Multivariate Anal., 11 (1981), 434-447.

[2] Jurek, Z. J., An integral representation of operator-selfdecomposable random variables, Bull. Acad. Polon. Sci. Sér. Sci. Math., 30 (1982), 385-393.

[ 3 ] — Limit distributions and one-parameter groups of linear operators on Banach spaces, J. Multivariate Anal., 13 (1983), 578-604.

[4] - The classes $L_{m}(Q)$ of probability measures on Banach spaces, Bull. Acad. Polon. Sci. Sér. Sci. Math., 31 (1983), 51-62.

[5] Jurek, Z. J. and Vervaat, W., An integral representation for self-decomposable Banach space valued random variables, Z. Wahrsch. Verw. Gebiete, 62 (1983), 247-262.

[6] Kumar, A. and Schreiber, B. M., Representation of certain infinitely divisible probability measures on Banach spaces, J. Multivariate Anal., 9 (1979), 288-303.

[ 7 ] Sato, K., Class $L$ of multivariate distributions and its subclasses, J. Multivariate Anal., 10 (1980), 207-232.

[ 8 ] — Absolute continuity of multivariate distributions of class $L$, J. Multivariate Anal., 12 (1982), 89-94.

[9] Sato, K. and Yamazato, M., Operator-selfdecomposable distributions as limit distributions of processes of Ornstein-Uhlenbeck type, Stochastic Process. Appl., 17 (1984), 73-100.

[10] - Stationary processes of Ornstein-Uhlenbeck type, Lecture Notes in Math., 1021 Springer, 1983, 541-551.

[11] Schmidt, K., Stable probability measures on $R^{\nu}$, Z. Wahrsch. Verw. Gebiete, 33 (1975), 19-31.

[12] Sharpe, M., Operator-stable probability distributions on vector groups, Trans. Amer. Math. Soc., 136 (1969), 51-65.

[13] Thu, N. V., A characterization of mixed-stable laws, Bull. Acad. Polon. Sci. Sér. Sci. Math., 27 (1979), 629-630.

[14] — Multiply self-decomposable probability measures on Banach spaces, Studia Math., 66 (1979), 161-175.

[15] Urbanik, K., Lévy's probability measures on Euclidean spaces, Studia Math., 44 (1972), 119-148.

[16] - Slowly varying sequences of random variables, Bull. Acad. Polon. Sci. Sér. Sci. Math. Astronom. Phys., 20 (1972), 679-682.

[17] _ Limit laws for sequences of normed sums satisfying some stability conditions, Multivariate Anal. III (ed. P. R. Krishnaiah), Academic Press, New York, 1973, 225-237.

[18] Wolfe, S. J., On a continuous analogue of the stochastic difference equation $X_{n}$ $=\rho X_{n-1}+B_{n}$, Stochastic Process. Appl., 12 (1982), 301-312.

[19] - A characterization of certain stochastic integrals, (Tenth Conference on Stochastic Processes and Their Applications, Contributed Papers), Stochastic Process. Appl., 12 (1982), 136. 
[20] Yamazato, M., Absolute continuity of operator-self-decomposable distributions on $R^{d}$, J. Multivariate Anal., 13 (1983), 550-560.

[21] — $O L$ distributions on Euclidean spaces, Teor. Verojatnost. i Primenen., 29 (1984), 3-18.

\section{Ken-iti Sato}

Department of Mathematics

College of General Education

Nagoya University

Nagoya 464

Japan

Makoto Yamazato

Department of Mathematics

Nagoya Institute of Technology

Nagoya 466

Japan 